\section{Determinação da infecção por Entamoeba histolytica em residentes da área metropolitana de Belém, Pará, Brasil, utilizando ensaio imunoenzimático (ELISA) para detecção de antígenos}

\author{
Determination of Entamoeba histolytica infection \\ in patients from Greater Metropolitan Belém, \\ Pará, Brazil, by enzyme-linked immunosorbent \\ assay (ELISA) for antigen detection
}

Mônica Cristina de Moraes Silva 1,2,3 Christiane do Socorro Pereira Monteiro 1 Bruna dos Anjos Veloso Araújo 1 Jacilene Valdevina Silva 1 Marinete Marins Póvoa 1

\footnotetext{
1 Seção de Parasitologia, Instituto Evandro Chagas, Belém, Brasil.

2 Fundação Instituto para o Desenvolvimento da Amazônia, Belém, Brasil.

3 Centro Universitário do Estado do Pará, Belém, Brasil.

Correspondência M. C. M. Silva. Laboratório de Enteroparasitoses, Seção de Parasitologia, Instituto Evandro Chagas. Rod. BR-316, $\mathrm{Km} 7$,

Ananindeua, $P A$ 67030-000, Brasil. monicamoraes@iec.pa.gov.br
}

\begin{abstract}
The epidemiological status of amebiasis has been reevaluated since Entamoeba histolytica (pathogenic) was considered a distinct species from Entamoeba dispar (non-pathogenic). We investigated $\mathrm{E}$. histolytica antigens in stool samples from residents of Belém, Pará State, Brazil, with commercially available enzyme-linked immunosorbent assay (E. histolytica Test, TechLab Inc., Blacksburg, USA). A total of 845 samples were analyzed, of which 248 were positive (29.35\%). E. histolytica infection was more frequent in the over-14-year age group (30.36\%) than in the 0-14-year group (28.28\%), but the difference was not statistically significant $(p<$ 0.05). Of all the samples, 334 were also submitted to parasitological methods (direct, Hoffman, and Faust et al.). There were discordant results between ELISA and parasitological methods in 83 samples (24.85\%), with more positive results using ELISA. Our results thus suggest that intestinal amebiasis is an important public health problem in Greater Metropolitan Belém.
\end{abstract}

Entamoeba histolytica; Immunodiagnosis; Amebiasis

\section{Introdução}

A amebíase, doença causada pelo protozoário Entamoeba histolytica, é considerada importante causa de morbi-mortalidade no homem. Esta parasitose apresenta ampla distribuição geográfica com alta prevalência em regiões tropicais, onde as condições de higiene e educação sanitária são consideradas deficientes. Segundo a Organização Mundial da Saúde (OMS) / Organização Pan-Americana da Saúde (OPAS) / Organização das Nações Unidas para a Educação, a Ciência e a Cultura (UNESCO) 1, o maior número de casos situa-se nos quatro continentes, com exceção da América Central e Oceania.

Vários inquéritos epidemiológicos têm sido realizados para estimar a incidência e a prevalência da amebíase no Brasil. Na maioria dos estudos, os métodos parasitológicos de fezes são os mais utilizados $2,3,4,5$, embora não sejam capazes de diferenciar E. histolytica (patogênica) de E. dispar, (não patogênica). Segundo Cunha et al. 6 , a prevalência da protozoose é bastante variável $(5,6 \%$ a $40,0 \%)$ nas diferentes regiões do país, assim como sua patogenicidade e virulência.

Técnicas capazes de identificar características antigênicas e genéticas específicas de $E$. histolytica têm sido desenvolvidas como novas ferramentas de diagnóstico, na tentativa de reduzir o tempo de duração dos testes e distinguir as duas espécies. $\mathrm{O}$ emprego do imunodiagnós- 
tico 7,8,9,10,11 e da biologia molecular 12,13,14,15,16,17 tem mostrado boa sensibilidade e especificidade. Devido ao alto custo, tais metodologias ainda não estão disponíveis para uso regular de diagnóstico, porém têm auxiliado em estudos epidemiológicos que resultam no conhecimento da prevalência da amebíase no mundo.

Esta investigação é uma continuidade do estudo anterior realizado por Póvoa et al. 18, e objetivou verificar a freqüência da infecção por E. histolytica utilizando um kit comercial para detecção de antígenos de E. histolytica. Um número maior de amostras fecais foi testado e outras áreas da região metropolitana de Belém, Pará, Brasil, foram incluídas neste estudo.

\section{Material e métodos}

\section{Amostra}

O estudo foi conduzido no período de junho de 1999 a abril de 2003, com indivíduos residentes na área metropolitana de Belém, de ambos os sexos e diferentes faixas etárias, atendidos no Laboratório de Enteroparasitoses do Instituto Evandro Chagas (IEC). A demanda espontânea foi composta por pacientes que apresentavam sinais ou sintomas sugestivos de enteroparasitoses e aqueles encaminhados pelos diferentes setores do IEC para diagnóstico complementar.

Os indivíduos, objeto de nosso estudo, ou seus responsáveis, foram esclarecidos sobre o conteúdo e os objetivos do projeto e assinaram o termo de consentimento informando a sua participação, conforme normas estabelecidas pelo Comitê de Ética em Pesquisa do IEC que aprovou este estudo.

\section{Coleta e análise parasitológica}

O período de coleta iniciou em junho de 1999, época em que a técnica de ELISA (ensaio imunoenzimático) foi implantada no IEC para diagnóstico da amebíase.

Apenas uma única amostra fecal foi fornecida por cada paciente, acondicionadas em frasco coletor sem conservante. No mesmo dia da coleta as amostras foram submetidas aos métodos direto (salina/Lugol), sedimentação espontânea e de Faust e colaboradores, e observadas em microscopia óptica (100x e 400x). Parte do material fecal foi guardado a $-20 \circ \mathrm{C}$ para posterior investigação por ELISA.
ELISA para detecção

de E. histolytica nas fezes

O kit ELISA utilizado neste estudo (E. histolytica Test, TechLab Inc., Blacksburg, Estados Unidos) foi desenvolvido para detecção rápida da infecção por E. histolytica em amostras fecais. O teste baseia-se na detecção da adesina (lectina inibidora de $\mathrm{N}$-acetil-D-galactosamina), presente na membrana do parasito e que faz a mediação da ligação dos trofozoítos às células da mucosa intestinal.

Os testes foram realizados de acordo com o protocolo do fabricante. Os espécimes fecais foram colocados em placas sensibilizadas com anticorpos policlonais (AcPo) que se ligam à adesina, seguida da adição do AcMo (conjugado à peroxidase) e incubação à temperatura ambiente por duas horas. A revelação é feita com a adição do substrato enzimático (tetrametilbenzidina) que desenvolverá cor na presença do complexo peroxidase-AcMo-adesina-AcPo. A densidade ótica foi medida em Espectofotômetro (Titerteck Multiskan ®Plus MK II) com filtro de luz de 450nm.

\section{Análise estatística}

As diferenças estatísticas entre as variáveis investigadas foram calculadas em programa estatístico BioEstat versão 2.0 19, empregando-se os testes do Qui-Quadrado $\left(\chi^{2}\right)$ e de McNemar, adotando nível de significância de $5 \%$.

\section{Resultados}

Neste estudo foram investigadas 845 amostras fecais, onde 495 correspondiam a pacientes pertencentes à faixa etária de 0-14 anos (grupo 1) e 350 acima de 14 anos (grupo 2). Utilizando-se o kit para detecção de antígenos de $E$. histolytica, obteve-se positividade em 248 das 845 amostras testadas, representando um porcentual de $29,35 \%$. A presença da infecção por E. histolytica foi maior no grupo 2 (30,36\%) que no grupo $1(28,28 \%)$, porém sem significância estatística $(p>0,05)$.

Trezentos e trinta e quatro amostras também foram analisadas quanto a presença de protozoários e helmintos, a partir do emprego de métodos parasitológicos de fezes. Foi encontrada infecção por E. histolytica/E. dispar em 84 (25,15\%) amostras, das quais 17 pertenciam a pacientes do grupo 1 e 67 a pacientes do grupo 2, sendo o patógeno mais prevalente nos dois grupos etários da amostra estudada. Trichuris trichiura foi o helminto mais freqüente, 
tendo sido encontrado em 8,38\% (28/334) das amostras, seguindo dos ancilostomídeos $(7,78 \%)$ e Ascaris lumbricoides (7,18\%). Cento e sete $(30,04 \%)$ indivíduos apresentaram infecções com mais de um protozoário/helminto, enquanto em $126(37,72 \%)$ não foi encontrado parasitismo.

Quando comparamos os resultados das 334 amostras analisadas por ambos os métodos (ELISA e coproscópicos) houve discordância nos resultados de 83 amostras $(24,85 \%)$, tendo ocorrido maior número de positivos no teste imunoenzimático $(\mathrm{p}<0,05)$ (Tabela 1$)$.

\section{Discussão}

Na região Amazônica, as precárias condições sócio-econômicas bem como a falta de saneamento básico na maioria das localidades desta região despertou o interesse de vários grupos de pesquisa acerca da prevalência das enteroparasitoses e vários inquéritos epidemiológicos têm sido realizados durante décadas.

No Pará, a amebíase é considerada um importante problema de saúde pública, visto que numerosos casos de formas invasivas, inclusive amebíase hepática, foram relatados por alguns autores 20,21,22,23,24,25. Assim, estudos epidemiológicos utilizando metodologias mais sensíveis para o diagnóstico da infecção por E. histolytica tornam-se necessários a fim de se evitar casos graves da doença.

A pesquisa de coproantígenos tem sido utilizada como diagnóstico alternativo da infecção, pois diferencia E. histolytica de E. dispar, reduz o tempo de execução dos testes, é de fácil execução e produz resultados satisfatórios. $\mathrm{O}$ kit ELISA tem se mostrado eficaz no diagnóstico da amebíase em diversos estudos epidemiológicos 18,26,27,28. Segundo Haque et al. 26, os resultados da detecção de coproantígenos por esse kit são comparáveis aos das técnicas isoenzimática e de biologia molecular, considerados padrão-ouro para diagnóstico da infecção.

No Brasil, esse kit tem sido utilizado por alguns grupos de pesquisa, porém os resultados observados até o momento mostram freqüências inferiores às observadas em Belém, tanto no estudo inicial realizado por Póvoa et al. 18, que encontrou positividade de $28,99 \%$, como neste estudo, cuja positividade foi de $29,35 \%$. Em Fortaleza (Ceará), Braga et al. 27,28 encontraram índices de positividade de 10,6\% 27 e 14,9\% 28 entre 564 e 735 amostras analisadas, respectivamente. Recentemente, Pinheiro et al. 29 , ao analisarem 59 amostras fecais de residentes na cidade de Macaparana (Pernambuco), não en-
Tabela 1

Resultados dos testes coproscópicos e ELISA (E. histolytica Test, TechLab Inc. Blacksburg, Estados Unidos) em 334 amostras testadas.

\begin{tabular}{|c|c|c|c|}
\hline \multirow[t]{2}{*}{ Coproscópico } & \multicolumn{2}{|c|}{ ELISA } & \multirow[t]{2}{*}{ Total } \\
\hline & Positivo & Negativo & \\
\hline Positivo & 43 & 31 & 74 \\
\hline Negativo & 52 & 208 & 260 \\
\hline Total & 95 & 239 & 334 \\
\hline
\end{tabular}

contraram positividade para E. histolytica. Estudos realizados em outras populações do mundo, revelam prevalências variáveis da doença.

O diagnóstico da amebíase intestinal pela pesquisa de cistos/trofozoítas nas fezes tem sido avaliado desde que E. histolytica foi considerada espécie distinta de E. dispar ${ }^{30}$. Resultados discordantes entre os métodos parasitológicos e outros métodos mais sensíveis têm sido freqüentemente observados.

Nossos achados demonstram elevada discordância $(24,85 \%)$ entre resultados dos testes coproscópico e ELISA. Tal discordância é esperada, pois os métodos parasitológicos são considerados de baixa sensibilidade não sendo capazes de diferenciar E. histolytica de E. dispar. Adicionalmente, a não diferenciação de E. histolytica/E. dispar de outras espécies de amebas pertencentes à classe Lobosea pode ter contribuído com os resultados falsos-negativos e falsos-positivos observados. Segundo Haque et al. 31 e Evangeloupolos et al. 32 a sensibilidade do método coproscópico está em torno de 60,0\%.

Dependendo da população estudada, os métodos coproscópicos podem subestimar ou superestimar a prevalência da doença. No Brasil, Braga et al. 27,28 encontraram uma subestimativa da infecção por E. histolytica na cidade de Fortaleza (Ceará). Por outro lado, estudos realizados em Pernambuco demonstram a presença apenas da E. dispar, considerada espécie não patogênica 29,33,34. Assim, nossos resultados sugerem que amebíase intestinal na região metropolitana de Belém é um importante problema de saúde pública.

De acordo com o estabelecido pela OMS 35, o tratamento da amebíase deverá ser adotado somente nos casos em que E. histolytica for especificamente confirmada. Além do método ELISA utilizado, outras técnicas podem ser empregadas como métodos alternativos ao exame parasitológico de fezes, dando maior confiabilidade ao diagnóstico da infecção. 


\section{Resumo}

O status epidemiológico da amebíase está sendo reavaliado desde que a Entamoeba histolytica (patogênica) foi considerada espécie distinta de Entamoeba dispar (não patogênica). Em nosso estudo, realizamos pesquisa de antígenos de $\mathrm{E}$. histolytica em amostras fecais de pacientes residentes na cidade de Belém, $\mathrm{Pa}$ rá, Brasil, utilizando ensaio imunoenzimático (E. histolytica Test, TechLab Inc., Blacksburg, Estados Unidos) disponível comercialmente. Foram analisadas 845 amostras, com positividade em 248 (29,35\%). A infecção por E. histolytica foi maior no grupo etário acima de 14 anos (30,36\%) que no grupo de 0-14 anos (28,28\%), porém sem significância estatística $(p<0,05)$. Do total de amostras analisadas, 334 foram também investigadas por métodos parasitológicos (direto, Hoffman e Faust et al.). Houve discordância nos resultados dos métodos ELISA e coproscópico em 83 amostras (24,85\%), com maior número de positivos no teste imunoenzimático. Assim, nossos resultados sugerem que a amebíase intestinal é um importante problema de saúde pública na Região Metropolitana de Belém.

Entamoeba histolytica; Imunodiagnóstico; Amebíase

\section{Colaboradores}

M. C. M. Silva elaborou o artigo e participou da execução da parte prática da pesquisa. C. S. P. Monteiro e B. A. V. Araújo executaram o diagnóstico imunológico. J. V. Silva executou o diagnóstico parasitológico. M. M. Póvoa supervisionou a elaboração do manuscrito e a execução dos diagnósticos.

\section{Referências}

1. World Health Organization/Pan-American Health Organization/United Nations Educational, Scientific and Cultural Organization. Proceedings of the XIII Seminar on Amebiasis. Mexico City, Mexico, January 29-31, 1997. Arch Med Res 1997; 28 Spec No:1-329.

2. Cardoso GS, Santana ADC, Aguiar CP. Prevalência e aspectos epidemiológicos da giardíase em creches no Município de Aracaju, SE, Brasil. Rev Soc Bras Med Trop 1995; 28:25-31.

3. Tavares-Dias M, Grandini AG. Prevalência e aspectos epidemiológicos de enteroparasitoses na população de São José da Bela Vista, São Paulo. Rev Soc Bras Med Trop 1999; 32:63-5.

4. Prado MS, Barreto ML, Strina A, Faria AS, Nobre AA, Jesus SR. Prevalência e intensidade da infecção por parasitas intestinais em crianças na idade escolar na Cidade de Salvador (Bahia, Brasil). Rev Soc Bras Med Trop 2001; 34:99-101.

5. Alves JR, Macedo HW, Ramos NA, Ferreira LF, Gonçalves MLC, Araújo A. Parasitoses intestinais em região semi-árida do nordeste do Brasil: resultados preliminares distintos das prevalências esperadas. Cad Saúde Pública 2003; 19:66770 .

6. Cunha AS, Castro L, Rocha P. Amebíase. In: Rocha PRS, Cunha AS, organizadores. Tópicos em gastroenterologia 2: gastroenterologia tropical. Rio de Janeiro: Editora Medsi; 1991. p. 287-316.

7. Zhang Y, Li E, Jackson TF, Zhang T, Gathiram V, Stanley SLJ. Use of a recombinate 170-kilodalton surface antigen of Entamoeba histolytica for serodiagnosis of amebiasis and identification of immunodominant domains of the native molecule. J Clin Microbiol 1992; 30:2788-92.

8. Abd-Alla MD, Jackson TF, Gathiram V, el-Hawey AM, Ravdin JI. Differentiation of pathogenic Entamoeba histolytica infections from nonpathogenic infections by detection of galactose-inhibitable adherence protein antigen in sera and feces. J Clin Microbiol 1993; 31:2845-50.

9. Haque R, Kress K, Jackson T, Lyerly D, Wilkins T, Petri Jr. WA. Diagnosis of pathogenic Entamoeba histolytica infection using a stool ELISA based on monoclonal antibodies to the galactose-specific adhesin. J Infect Dis 1993; 167:247-9.

10. Braga LL, Lima AAM, Sears CL, Newman RD, Wuhib T, Paiva CA. Seroepidemiology of Entamoeba histolytica in slum northeast Brazil. Am J Trop Med Hyg 1996; 55:693-7.

11. Abd-Alla MD, Ravdin JI. Diagnosis of amoebic colitis by antigen capture ELISA in patients presenting acute diarrhoea in Cairo, Egypt. Trop Med Intern Health 2002; 7:365-70.

12. Tannich E, Burchard GD. Differentiation of pathogenic from non pathogenic Entamoeba histolytica by restriction fragment analysis gene amplified in vitro. J Clin Microbiol 1991; 28:250-5.

13. Acuna-Soto R, Samuelson J, De Girolami P, Zarate M, Mollan-Velasco F, Schooinick G, et al. Application of the polimerase chain reaction to the epidemiology of pathogenic and nonpathogenic Entamoeba histolytica. Am J Trop Med Hyg 1993; 48:58-70. 
14. Katzwinkel-Wladarsch S, Loscher T, Rinder H. Direct amplification and differentiation of pathogenic and nonpathogenic Entamoeba histolytica DNA from stool specimens. Am J Trop Med Hyg 1994; 51:115-8.

15. Britten D, Wilson SM, McNerney R, Moody AH, Chiodini PL, Ackers JP. An improved colorimetric PCR-based method for detection and differentiation of Entamoeba histolytica and Entamoeba dispar in feces. J Clin Microbiol 1997; 35:1108-11.

16. Aguirre A, Molina S, Blotkamp C, Verveij J, Vinuesa T, Valls ME, et al. Diagnosis of Entamoeba histolytica and Entamoeba dispar in clinical specimens by PCR-SHELA. Arch Med Res 1997; 28:282-4.

17. Verweij JJ, Blotkamp J, Brienen EA, Aguirre A, Polderman AM. Differentiation of Entamoeba histolytica and Entamoeba dispar cysts using polymerase chain reaction on DNA isolated from faeces with spin columns. Eur J Clin Microbiol Infect Dis 2000; 19:358-61.

18. Póvoa MM, Arruda JE, Silva MC, Bichara CN, Esteves P, Gabbay YB, et al. Diagnóstico de amebíase intestinal utilizando métodos coproscópicos e imunológicos em amostra da população da área metropolitana de Belém, Pará, Brasil. Cad Saúde Pública 2000; 16:843-6.

19. Ayres M, Ayres Jr. M, Ayres DL, Santos AS, organizadores. BioEstat: aplicações estatísticas nas áreas das ciências biológicas e médica. Manaus: Sociedade Civil Mamirauá/Ministério da Ciência e Tecnologia/Conselho Nacional de Desenvolvimento Científico e Tecnológico; 2000.

20. Araújo R. Lesões amebianas do grosso intestino: estudo anátomo-patológico. Rev Cienc Biol 1963; 1:103-24.

21. Rodrigues Filho A, Leite JM, Vianna C, Araújo R, Maneschy L, Soares EC. Abscesso amebiano do fígado: estudo clínico e anátomo-patológico de 13 casos. Rev Cienc Biol 1963; 1:162-76.

22. Chaves LCL. Abscesso amebiano do fígado [Dissertação de Mestrado]. Rio de Janeiro: Universidade Federal do Rio de Janeiro; 1987.

23. Araújo R, Cruz EM, Araújo MTF. Estudo anátomopatológico da amebíase em Belém do Pará. In: Anais do XXIV Congresso da Sociedade Brasileira de Medicina Tropical. Manaus: Sociedade Brasileira de Medicina Tropical; 1998. p. 141.

24. Silva EB, Neto LC, Guerra ALS, Gomes GAR. Estudo clínico de abscesso hepático em crianças. In: Anais do XXIV Congresso da Sociedade Brasileira de Medicina Tropical. Manaus: Sociedade Brasileira de Medicina Tropical; 1998. p. 141.
25. Salles JM, Moraes LA, Salles MC. Hepatic amebiasis. Braz J Infect Dis 2003; 7:96-110.

26. Haque R, Ali IKM, Akther S, Petri Jr. WA. Comparasion of PCR, isoenzyme analysis, and antigen detection for diagnosis of Entamoeba histolytica infection. J Clin Microbiol 1998; 36:449-53.

27. Braga LL, Mendonça Y, Paiva CA, Sales A, Cavalcante ALM, Mann BJ. Seropositivity for and intestinal colonization with Entamoeba histolytica and Entamoeba dispar in individuals in Northeastern Brazil. J Clin Microbiol 1998; 36:3044-5.

28. Braga LL, Gomes ML, Silva MW, Paiva C, Sales A, Mann BJ. Entamoeba histolytica and Entamoeba dispar infections as detected by monoclonal antibody in an urban slum in Fortaleza, Northeastern Brazil. Rev Soc Bras Med Trop 2001; 34:467-71.

29. Pinheiro SMB, Carneiro RM, Aca IS, Irmão JI, Morais MA, Coimbra MRM, et al. Determination of the prevalence of Entamoeba histolytica and E. dispar in the Pernambuco state of the northeastern Brazil by a polymerase chain reaction. Am J Trop Med Hyg 2004; 70:221-4.

30. Diamond L, Clark CG. A redescription of Entamoeba histolytica Schaudinn, 1903 (emended Walker, 1911) separating it from Entamoeba dispar Brumpt, 1925. J Eucaryot Microbiol 1993; 40:340-4.

31. Haque R, Faruque ASG, Hahn P, Lyerly DM, Petri Jr. WA. Entamoeba histolytica and E. dispar infection in children Bangladesh. J Infect Dis 1997; 175:734-6.

32. Evangeloupolos A, Legakis N, Vaikalis N. Microscopy, PCR and ELISA applied to the epidemiology of amoebiasis in Greece. Parasitol Int 2001; 50:185-9.

33. Tachibana H, Kobayashi S, Paz KC, Aca IS, Tateno $\mathrm{S}$, Ihara S. Analysis of pathogenicity by restriction-endonuclease digestion of amplified genomic DNA of Entamoeba histolytica isolated in Pernambuco, Brazil. Parasitol Res 1992; 78:433-6.

34. Aca IS, Kobayashi S, Carvalho LB, Tateno S, Takeuchi T. Prevalence and pathogenicity of Entamoeba histolytica in three different regions of Pernambuco, northeast Brazil. Rev Inst Med Trop São Paulo 1994; 36:519-24.

35. World Health Organization. Entamoeba taxonomy. Bull World Health Organ 1997; 75:291-2.

Recebido em 30/Jul/2004

Versão final reapresentada em 10/Jan/2005

Aprovado em 13/Jan/2005 